Portland State University

PDXScholar

Electrical and Computer Engineering Faculty

Publications and Presentations

10-1-2002

Recurring Beams in Hollow Metal Waveguides:

Paraxial Approximation

Lee W. Casperson

Portland State University

Follow this and additional works at: https://pdxscholar.library.pdx.edu/ece_fac

Part of the Electrical and Computer Engineering Commons

Let us know how access to this document benefits you.

Citation Details

Lee W. Casperson, "Recurring Beams in Hollow Metal Waveguides: Paraxial Approximation," Appl. Opt. 41, 6410-6415 (2002).

This Article is brought to you for free and open access. It has been accepted for inclusion in Electrical and Computer Engineering Faculty Publications and Presentations by an authorized administrator of PDXScholar. Please contact us if we can make this document more accessible: pdxscholar@pdx.edu. 


\title{
Recurring beams in hollow metal waveguides: paraxial approximation
}

\author{
Lee W. Casperson
}

\begin{abstract}
For optical and near-optical applications in electromagnetics, the directed propagation of waves in free space and in lenslike media is often in the Cartesian form of Gaussian or more general Hermitesinusoidal-Gaussian beams. It has been shown that recurring (rather than continuing) forms of such beams are possible in the paraxial approximation for certain hollow metal waveguides, in which multiple reflections from the waveguide walls may occur. Limitations on this recurrence behavior implicit in use of the paraxial approximation are considered here, and estimates are obtained for the maximum propagation distance before the onset of significant distortion of the recurring beams. (C) 2002 Optical Society of America
\end{abstract}

OCIS codes: $230.0230,230.7370,230.7390,350.5500$.

\section{Introduction}

Gaussian beams and related higher-order Hermitesinusoidal-Gaussian beams are widely employed for the unguided propagation of electromagnetic waves. The shape-invariance and the optimum collimation and focusing properties of such beams give them important advantages for practical applications. Also, these beams are naturally generated in many laser systems. However, for some environments and wavelengths unguided propagation is not practical, and one may be led to seek transparent waveguiding media. Such media have been developed, including lenslike materials that are capable of transmitting Gaussian beams. Nevertheless, there remain important spectral regions where satisfactory and economical dielectric waveguiding materials are not available. In such circumstances one may employ narrower versions of the microwave-waveguiding standby, consisting of hollow metal tubes. Such waveguides have already been widely used at infrared and optical frequencies, for example, for propagation of the important $10.6-\mu \mathrm{m}$ radiation fields from $\mathrm{CO}_{2}$ lasers. ${ }^{1-20}$

A disadvantage of metal waveguides for the prop-

L. W. Casperson (lcaspers@ee.pdx.edu) is with the Departments of Electrical and Computer Engineering and of Physics, Portland State University, P.O. Box 751, Portland, Oregon 97207-0751.

Received 5 February 2002; revised manuscript received 2 July 2002.

0003-6935/02/306410-06\$15.00/0

(C) 2002 Optical Society of America agation of electromagnetic fields is that they do not support the propagation of shape-invariant or continuing Gaussian beams. As a result, the coupling of Gaussian beams into or out of such waveguides can be relatively inefficient. However, it has been shown that recurring Gaussian beams may be possible in hollow metal waveguides, even though continuing Gaussian beams are not. ${ }^{21,22}$ That is, Gaussian field distributions are not possible at all planes along a metal waveguide, but they may recur periodically at certain well-defined and easily calculable distances. With appropriate design, two of these planes of Gaussian-beam recurrence can correspond to the entrance and the exit of the waveguide. Then the waveguide fields will have coupled efficiently to free-space Gaussian input and output beams, and this possibility was demonstrated in a recent experiment. ${ }^{23}$

The recurring beam fields that have just been described were obtained in Ref. 22 as solutions of the paraxial wave equation. In fact, the standard freely propagating Gaussian beams are obtained only as solutions of the paraxial wave equation, and without that approximation the beam fields do not possess their simple and familiar shape invariance. The wide acceptance of Gaussian beams for the propagation of electromagnetic beam fields would seem to suggest that the paraxial approximation usually must be valid. More specifically, the analysis that yields recurring Gaussian beams in hollow metal waveguides is based on solutions of the paraxial equation, and it might be imagined that these solu- 


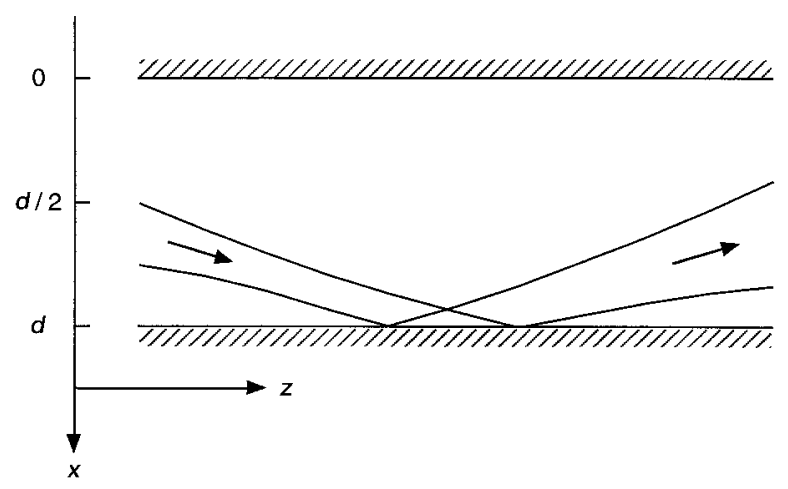

Fig. 1. Schematic representation of an off-axis Gaussian beam undergoing diffraction and reflection from the flat parallel surfaces of a waveguide. The coordinate system used in the analysis is also shown.

tions would be valid for many waveguide configurations.

The most straightforward way to explore the limitations of the paraxial approximation is to recognize that the recurring beams must be representable as a superposition of the basic waveguide modes. ${ }^{24,25}$ The advantage of this approach is that the waveguide modes can be obtained analytically either with or without the paraxial approximation. A brief paraxial treatment was given in Ref. 22, and the corresponding exact nonparaxial procedures are developed here. A comparison of the solutions obtained in these two ways reveals the consequences of the paraxial approximation for beam recurrence. It is possible to obtain an analytical estimate of the distance after which the recurrences cease to be useful reproductions of the initial input-field distribution.

A brief review of the metal waveguide modes based on the paraxial approximation is included in Section 2 , and the corresponding derivation without the paraxial approximation is discussed in Section 3. The results are applied to find estimates of the distance over which the paraxial approximation and its implications for beam recurrence are reliable. In general, this distance decreases as the transverse dimensions of the waveguide decrease and as the complexity of the waveguide field increases. An example is given to indicate the distortions that occur when the paraxial approximation ceases to be valid.

\section{Paraxial Waveguide Modes}

One of the basic geometries to be considered here consists of a diffracting beam injected between two parallel reflecting metal surfaces. This beam is not necessarily introduced at the center of the waveguide input plane, and it might also be propagating in part toward or away from one of the waveguide sides. This arrangement is shown in Fig. 1, and the coordinate system to be employed is also shown in the figure. Owing to diffraction and misalignment, the beam interacts with the waveguide surfaces over extended distances, and these surfaces are assumed to be highly reflecting and flat.
For the case of nearly plane waves in a medium in which the changes in permittivity and permeability are small, the dominant transverse Cartesian field components are governed by the wave equation

$$
\nabla^{2} \mathbf{E}^{\prime}(x, y, z)+k^{2}(x, y, z) \mathbf{E}^{\prime}(x, y, z)=0,
$$

where $\mathbf{E}^{\prime}$ is the complex amplitude of the electric field and $k$ is the potentially complex spatially dependent wave number. While Eq. (1) governs the dominant transverse components, the weak $z$ components of the fields may be found at any location from the transverse components by means of the MaxwellHeaviside equations. ${ }^{26}$

For applications of interest here it is assumed that the propagation medium within the waveguide is essentially a uniform dielectric, and thus $k$ in Eq. (1) is considered to be a real constant. For consistency with Ref. 22 , this constant will be represented as $\beta_{0}$. Furthermore, because of the separability of the wave equation for a uniform medium, no particular insight is gained by retaining the propagation effects in both of the transverse directions. Thus we assume at the outset that there are no field variations in the $y$ direction. Also, for the dominant and most-used mode in a practical rectangular waveguide, the fields do not vary in one of the transverse directions. Thus the following analysis sometimes may be applicable without change for a rectangular waveguide when the tangentially polarized fields are treated.

As indicated above, we will be comparing the implications of certain waveguide solutions that are obtained with and without the paraxial approximation. To begin with, the paraxial approximation will be employed. Thus for a linearly polarized wave that propagates primarily in the $z$ direction, a useful substitution is

$$
\mathrm{E}^{\prime}(x, y, z)=A(x, z) \exp \left(-i \beta_{0} z\right),
$$

where $A$ is a complex amplitude function. With this substitution Eq. (1) reduces to the paraxial wave equation

$$
\frac{\partial^{2} A}{\partial x^{2}}-2 i \beta_{0} \frac{\partial A}{\partial z}=0
$$

where $A$ is assumed to vary so slowly with $z$ that its second derivative can be neglected.

In Ref. 22 the propagation of electromagnetic fields in waveguides was interpreted in terms of a superposition of fundamental Gaussian beam solutions of Eq. (3). As an illustration, Fig. 2 shows a series of transverse intensity profiles for a normalized Gaussian beam that is undergoing diffraction and reflection from the flat waveguide surfaces located at the positions $x=0, d$. The beam in this case is polarized parallel to the surfaces and enters along the center axis of the waveguide with its waist at the input plane. The waist spot size is $w_{0} / d=0.2$, and the propagation distance between successive profiles is $z / z_{0}=0.1$ (ten plots per Rayleigh length). The intensity spreads into two symmetrical peaks and then 


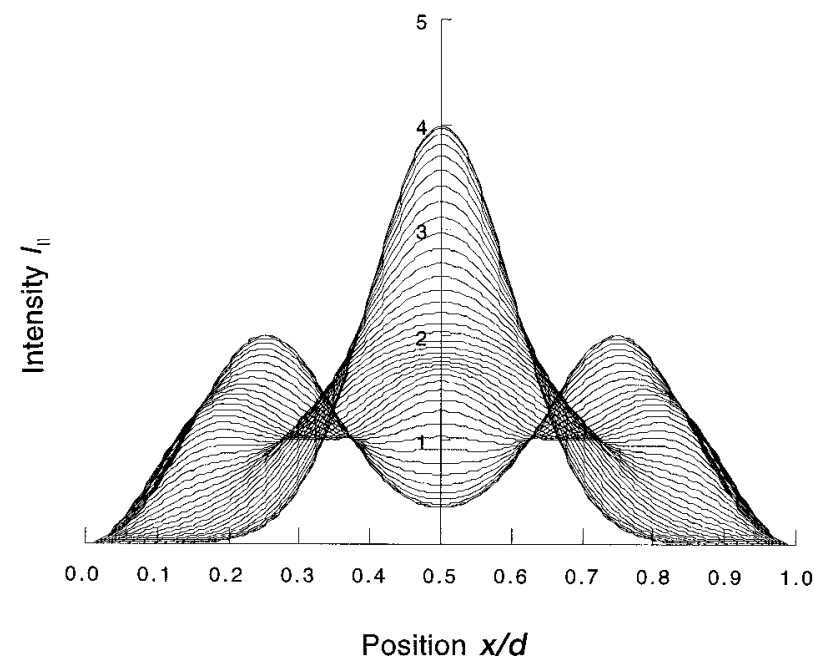

Fig. 2. Series of transverse intensity profiles of a normalized on-axis paraxial Gaussian beam interacting with flat waveguide surfaces located at the positions $x / d=0,1$. The beam is polarized parallel to the surfaces, the waist spot size is $w_{0} / d=0.2$, and the propagation distance between successive profiles is $z / z_{0}=0.1$ (ten plots per Rayleigh length). The original near-Gaussian profile recurs after approximately eight Rayleigh lengths.

returns to its initial near-Gaussian shape after approximately eight Rayleigh lengths $\left(z_{0}=\pi w_{0}^{2} / \lambda\right)$. To make this illustration more quantitative, we may imagine that the waveguide width is $5 \mathrm{~mm}$, and the input beam is from a $\mathrm{CO}_{2}$ laser with a wavelength $\lambda=$ $10.6 \mu \mathrm{m}$ and a spot size $w_{0}=1 \mathrm{~mm}$. Thus the Rayleigh length would be $z_{0}=\pi\left(10^{-3}\right)^{2} / 10.6 \times$ $10^{-6}=0.3 \mathrm{~m}$. As required by the boundary conditions for parallel polarization, the intensity in this example always remains zero at the reflecting surfaces.

Although the Gaussian beam-summation method can be quite efficient, an alternative analysis based on an expansion of the input beam in a set of waveguide eigenmodes can provide a more direct perspective on the recurring nature of the fields. The waveguide mode approach was discussed briefly in Ref. 22, and some of those results are reproduced here as a basis for the nonparaxial generalizations in the following section. It is assumed that the field amplitude $A(x, z)$ in Eq. (3) can be written in the form

$$
A(x, z)=B(x) \exp \left(-i \gamma_{\mathrm{par}} z\right),
$$

where, for the propagating modes, $\gamma_{p a r}$ is a real quantity representing the modal correction to the planewave phase in the paraxial approximation. With this substitution, the transverse part of the paraxial wave equation is

$$
\frac{\mathrm{d}^{2} B}{\mathrm{~d} x^{2}}-2 \beta_{0} \gamma_{\mathrm{par}} B=0 .
$$

If the $x$ coordinate has its origin at one of the waveguide surfaces as in Fig. 1, it is natural to express the solutions of Eq. (5) in the form

$$
B(x)=a \sin k_{x} x+b \cos k_{x} x .
$$

When Eq. (6) is substituted into Eq. (5), one obtains the characteristic equation

$$
k_{x}^{2}+2 \beta_{0} \gamma_{\mathrm{par}}=0 .
$$

If the electric field is polarized parallel to the waveguide surfaces, the amplitude must go approximately to zero at those surfaces. For a waveguide spacing of $d$, the field solution from Eq. (6) is therefore of the form

$$
B(x)=a \sin k_{x} x,
$$

where the transverse propagation constant $k_{x}$ must satisfy the condition

$$
k_{x} d=m \pi,
$$

with the mode index given by $m=1,2,3, \ldots$. Equations (7) and (9) may be combined to obtain the modal phase correction

$$
\gamma_{\mathrm{par}} z=-\frac{1}{2 \beta_{0}} \frac{m^{2} \pi^{2}}{d^{2}} z
$$

An arbitrary input field distribution can be expanded in terms of the waveguide modes. In the more general cases one may expect a nonzero amplitude for many of the lower-order waveguide modes in such an expansion. As discussed in Ref. 22, the phase differences between these mode components will first recur at the distance

$$
z=\frac{4 \beta_{0} d^{2}}{\pi},
$$

and this recurrence of the phase differences would also correspond to a recurrence of the overall field distribution. For completeness we mention that for this parallel polarization a symmetric input field would include only modal components for which the mode index $m$ in Eq. (9) is an odd integer, and one finds that consequently a symmetric field would recur at the shorter distance

$$
z=\frac{\beta_{0} d^{2}}{2 \pi} .
$$

Similar results are obtained for the case that the field is polarized perpendicular to the waveguide surfaces.

\section{Nonparaxial Corrections}

The preceding discussion has concerned recurring beam solutions with a paraxial model for hollow metal waveguides. However, the modes of such a waveguide can also be obtained analytically without use of the paraxial approximation. For obtaining these exact results, we begin again with Eq. (1) with the propagation constant $k$ of the medium replaced by $\beta_{0}$ for a real dielectric. For a linearly polarized wave that propagates primarily in the $z$ direction a useful substitution is

$$
\mathrm{E}^{\prime}(x, y, z)=B(x) \exp \left(-i \beta_{z} z\right),
$$


where $B$ is a new complex amplitude function and $\beta_{z}$ is the actual propagation constant for the field in the waveguide. With this substitution Eq. (1) reduces to the ordinary differential equation

$$
\frac{\mathrm{d}^{2} B}{\mathrm{~d} x^{2}}+\left({\beta_{0}}^{2}-\beta_{z}{ }^{2}\right) B=0 .
$$

As in the paraxial case, it will be assumed that the solutions of Eq. (14) are in the form of Eq. (6), and thus the characteristic equation is now

$$
k_{x}{ }^{2}+\left(\beta_{z}{ }^{2}-\beta_{0}{ }^{2}\right)=0 .
$$

If the electric field is polarized parallel to the waveguide surfaces, the amplitude must go approximately to zero at those surfaces. For a waveguide spacing of $d$, the transverse propagation constant $k_{x}$ must again satisfy Eq. (9). Combining Eqs. (9) and (15), one finds that the total phase shift for propagation a distance $z$ can be written

$$
\beta_{z} z=\left[\beta_{0}{ }^{2}-(m \pi / d)^{2}\right]^{1 / 2} z .
$$

In the paraxial treatment of the previous section, the product $\gamma_{\mathrm{par}} z$ represented the modal correction to the plane-wave phase. In a similar way we now use Eq. (16) to introduce the exact nonparaxial modal phase correction:

$$
\begin{aligned}
\gamma_{\mathrm{ex}} z & =\left\{\left[\beta_{0}^{2}-(m \pi / d)^{2}\right]^{1 / 2}-\beta_{0}\right\} z \\
& =\left\{\left[1-\left(m \pi / \beta_{0} d\right)^{2}\right]^{1 / 2}-1\right\} \beta_{0} z .
\end{aligned}
$$

For short wavelengths and wide waveguides it might be anticipated that the exact expression in Eq. (17) could be simplified without substantial errors. If the term $\left(m \pi / \beta_{0} d\right)^{2}$ is assumed to be small compared with unity, Eq. (17) can be written to the second order in this quantity as

$$
\begin{aligned}
\gamma_{\mathrm{ex}} z_{s} & \approx\left[-\frac{1}{2}\left(\frac{m \pi}{\beta_{0} d}\right)^{2}-\frac{1}{8}\left(\frac{m \pi}{\beta_{0} d}\right)^{4}\right] \beta_{0} z_{s} \\
& =\left[-\frac{1}{2}\left(\frac{m \pi}{\beta_{0} d}\right)^{2}-\frac{1}{8}\left(\frac{m \pi}{\beta_{0} d}\right)^{4}\right] \frac{\beta_{0}^{2} d^{2}}{2 \pi} s,
\end{aligned}
$$

where the distance $z$ has been replaced by $z_{s}$, which represents $s$ times the paraxial recurrence distance for a symmetric parallel-polarized mode, as given in Eq. (12). The propagation constant $\beta_{0}$ is related to the wavelength $\lambda$ and the index of refraction $n$ of the dielectric in the waveguide by $\beta_{0}=2 \pi n / \lambda$. With this substitution in Eq. (18), the phase delay to paraxial recurrence number $s$ can be written as

$$
\begin{aligned}
\gamma_{\mathrm{ex}} z_{s} & =\left[-\frac{m^{2} \pi}{4}-\frac{m^{4} \pi}{64}\left(\frac{\lambda}{n d}\right)^{2}\right] s \\
& =\gamma_{\mathrm{par}} z_{s}-\frac{m^{4} \pi}{64}\left(\frac{\lambda}{n d}\right)^{2} s,
\end{aligned}
$$

where Eqs. (10) and (12) have been used to reintroduce the paraxial phase delay.

Equation (19) provides an indication of the leading phase errors resulting from the use of the paraxial approximation at the recurrences of a symmetric parallel-polarized beam in a metal waveguide of width $d$. Because the beam was assumed to be symmetric, only odd values of the index $m$ are to be considered. The beam at the paraxial recurrence position will resemble the initial beam only if the correction term in Eq. (19) is sufficiently small. As an approximate smallness criterion, one might write

$$
\frac{m^{4} \pi}{64}\left(\frac{\lambda}{n d}\right)^{2} s<\frac{\pi}{4} .
$$

If the medium in the waveguide is free space $(n=1)$, Eq. (20) can be written as

$$
m<2(d / \lambda)^{1 / 2} s^{-1 / 4} .
$$

An example in Ref. 22 involved a $\mathrm{CO}_{2}$ laser beam at $10.6-\mu \mathrm{m}$ wavelength propagating through a waveguide of width $0.5 \mathrm{~mm}$. With these values in Eq. (21), the beam at the first recurrence $(s=1)$ would be a good representation of the input beam even if $m$ values up to approximately 13 were used in the input-beam representation, and from Eq. (12) this recurrence distance is $23.6 \mathrm{~mm}$.

The maximum usable distance is evidently a sensitive function of the spacing $d$ between the waveguide surfaces. It follows from Eq. (20) that for a given mode index $m$ and wavelength $\lambda$ the maximum recurrence order $s$ is proportional to the square of the spacing $d$. At the same time, from Eq. (12) the propagation distance for each recurrence also increases quadratically with $d$, so the overall usable length increases rapidly with the waveguide spacing.

An $m$ value of 13 , as mentioned in the example above, might be far more than is needed to obtain an adequate beam description. It is clear from Eq. (21) that if sixteen recurrences were desirable $(s=16)$, the maximum $m$ value for describing the beam would be reduced by a factor of two $\left(16^{1 / 4}=2\right)$ to about seven. For the $\mathrm{CO}_{2}$ laser just discussed, the sixteenth recurrence would occur in a distance of approximately $z=23.6 \mathrm{~mm} \times 16 \cong 380 \mathrm{~mm}$. Evidently, the distance over which high-fidelity beam recurrences are possible is also a sensitive function of the maximum mode index $m$ needed to represent the beam.

It is possible to estimate the highest mode index $m$ needed in the beam expansion and also to calculate the profile of the recurring beam as it degrades owing to a failure of the paraxial approximation. Equation (8) represents the transverse field variation of a single waveguide mode when the field is polarized parallel to the waveguide surfaces. It follows that an arbitrary input beam should be expandable in such waveguide modes in the form

$$
A(x, 0)=\sum_{m=1}^{\infty} a_{m} \sin (m \pi x / d) .
$$


This expression can be inverted to obtain the expansion coefficients in terms of the input-field distribution

$$
a_{m}=\frac{2}{d} \int_{0}^{d} A(x, 0) \sin \left(\frac{m \pi x}{d}\right) \mathrm{d} x .
$$

For some input fields the expansion coefficients can be approximated analytically. For the simplest and perhaps most important case of a centered Gaussian beam waist at the input, the normalized field distribution can be written as $^{22}$

$$
A(x, 0)=\left[\frac{(2 / \pi)^{1 / 2} d}{w_{0}}\right]^{1 / 2} \exp \left[-\left(\frac{x-d / 2}{w_{0}}\right)^{2}\right] .
$$

For this input Eq. (23) is

$$
\begin{aligned}
a_{m}= & \frac{2}{d}\left[\frac{(2 / \pi)^{1 / 2} d}{w_{0}}\right]^{1 / 2} \int_{0}^{d} \exp \left[-\left(\frac{x-d / 2}{w_{0}}\right)^{2}\right] \\
& \times \sin \left(\frac{m \pi x}{d}\right) \mathrm{d} x .
\end{aligned}
$$

In order for this waveguide to support a recurring Gaussian field, it is necessary that the input spot size $w_{0}$ be substantially smaller than the waveguide width $d$. In this case the integration interval can be extended to infinity with negligible error, and Eq. (25) is replaced by

$$
\begin{aligned}
a_{m}= & \frac{2}{d}\left[\frac{(2 / \pi)^{1 / 2} d}{w_{0}}\right]^{1 / 2} \int_{-\infty}^{\infty} \exp \left[-\left(\frac{x-d / 2}{w_{0}}\right)^{2}\right] \\
& \times \sin \left(\frac{m \pi x}{d}\right) \mathrm{d} x
\end{aligned}
$$

But this integral can be performed analytically, and the result is ${ }^{27}$

$$
a_{m}=2\left[\frac{(2 \pi)^{1 / 2} w_{0}}{d}\right]^{1 / 2} \exp \left[-\left(\frac{m \pi w_{0}}{2 d}\right)^{2}\right](-1)^{(\mathrm{m}-1) / 2},
$$

provided that $m$ is odd, while $a_{m}=0$ for $m$ even. This result shows that the expansion coefficients become very small for $\mathrm{m} \gg d / w_{0}$. For the illustrations in Ref. 22 , for example, $d / w_{0}$ was equal to 5 , so expansion terms with $m$ values much greater than 5 would contribute little to the field at any reference plane. Accordingly, from Eq. (21) dozens of recurrences should be possible before significant deterioration of the beam would occur.

Once the expansion coefficients are known, the actual field distribution at the recurrence planes can be calculated. The phase discrepancy at any plane $z$ for mode number $m$ can be found by subtracting $\gamma_{\text {par }} z$ of Eq. (10) from $\gamma_{\mathrm{ex}} z$ of Eq. (17):

$$
\begin{aligned}
\Delta \gamma_{m} z= & \left\{\left[1-(m \lambda / 2 n d)^{2}\right]^{1 / 2}+(1 / 2)\right. \\
& \left.\times(m \lambda / 2 n d)^{2}-1\right\}(2 \pi n / \lambda) z .
\end{aligned}
$$

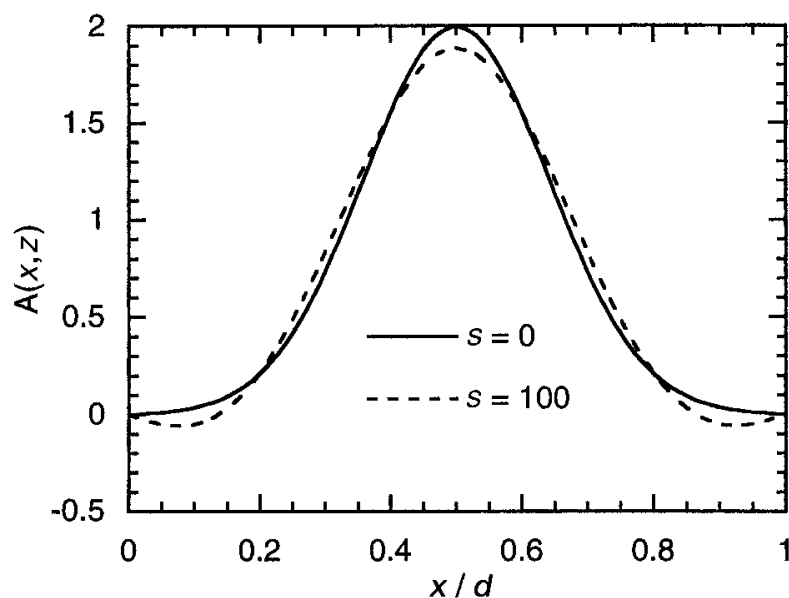

Fig. 3. Transverse amplitude profiles of a normalized on-axis Gaussian beam at recurrences $s=0$ and $s=100$, represented as a summation of nonparaxial waveguide modes.

With Eq. (12) for the parallel-polarization paraxial recurrence distance, the phase error at recurrence $s$ can be written

$$
\begin{aligned}
\Delta \gamma_{m} z_{s}= & \left\{\left[1-(m \lambda / 2 n d)^{2}\right]^{1 / 2}+(1 / 2)\right. \\
& \left.\times(m \lambda / 2 n d)^{2}-1\right\}\left(2 \pi n^{2} d^{2} / \lambda^{2}\right) s .
\end{aligned}
$$

With Eqs. (22), (27), and (29) the instantaneous field distribution at paraxial recurrence plane $s$ can be written

$$
\begin{aligned}
A\left(x, z_{s}\right)= & \sum_{m \text { odd }} 2\left[\frac{(2 \pi)^{1 / 2} w_{0}}{d}\right]^{1 / 2} \exp \left[-\left(\frac{m \pi w_{0}}{2 d}\right)^{2}\right] \\
& \times(-1)^{(m-1) / 2} \sin \left(\frac{m \pi x}{d}\right) \cos \left(\Delta \gamma_{m} z_{s}\right) .
\end{aligned}
$$

As an illustration, the field distribution is plotted in Fig. 3 for the dimensions and wavelength of a $\mathrm{CO}_{2}$ laser waveguide, as mentioned above. Specifically, the field is calculated at the plane $s=0$ (input) and at the plane $s=100$. The $s=0$ field is equivalent to the original input Gaussian, which is a verification of the expansion process, whereas the field at a sufficiently large distance is significantly distorted. In general, high-fidelity recurrences of Gaussian beams in suitably designed metal waveguides are possible, at least in principle, over kilometer distances. It should be mentioned that the above calculations have been carried out for the case that the field is symmetric and polarized parallel to the waveguide surfaces. Similar results can be obtained for the case that the field is asymmetric or is polarized perpendicular to these surfaces, and related calculations should be possible for other types of waveguide.

A related question for the practical use of beam recurrence is the length tolerance required for this effect. Specifically, one might like to know by how much the waveguide length can differ from the exact length to a recurrence plane. This question can be considered in the paraxial approximation; when this approximation has failed, no high quality recurrences 
can be expected. For a Gaussian input beam in the paraxial approximation, the answer is straightforward. Since the recurrences will also be Gaussians, a reasonable estimate of the length tolerance is the Rayleigh length $z_{0}$.

\section{Conclusion}

It has been shown that recurring Gaussian beams are solutions of the paraxial wave equation for propagation in certain types of hollow metal waveguide. Similar recurrences also occur for more-complicated field distributions. The purpose of this study has been to see under what conditions these recurrences would be modified if the paraxial approximation were not employed. It has been found that without the paraxial approximation there must be a gradual degrading of the mode profile at the successive recurrences. However, for typical profiles that include mainly low-order waveguide mode components in not-too-narrow waveguides, the paraxial approximation introduces only small errors, and many clear recurrences should be possible. Thus these recurring field solutions may find practical applications for the guided transmission of desired beam profiles over long distances.

\section{References}

1. E. Garmire, T. McMahon, and M. Bass, "Propagation of infrared light in flexible hollow waveguides," Appl. Opt. 15, 145-150 (1976).

2. E. Garmire, "Propagation of IR light in flexible hollow waveguides: further discussion," Appl. Opt. 15, 3037-3039 (1976).

3. E. Garmire, T. McMahon, and M. Bass, "Low-loss optical transmission through bent hollow metal waveguides," Appl. Phys. Lett. 31, 92-94 (1977).

4. E. Garmire, T. McMahon, and M. Bass, "Measurements of propagation in flexible infrared transmissive (FIT) waveguides,” IEEE J. Quantum Electron. QE-13, 21-22 (1977).

5. T. Matsushima, I. Yamauchi, and T. Sueta, "Flexible infraredtransmissive plastic waveguides coated with evaporated aluminum,” Jpn. J. Appl. Phys. 20, 1345-1346 (1981).

6. J. Gombert and M. Gazard, "Attenuation characteristics of a planar dielectric coated metallic waveguide for $10.6-\mu \mathrm{m}$ radiation," Opt. Commun. 58, 307-310 (1986).

7. M. Miyagi and S. Karasawa, "A comparative study of rectangular and circular dielectric-coated metallic waveguides for $\mathrm{CO}_{2}$ laser light: theory," Opt. Commun. 68, 18-20 (1988).

8. S. V. Azizbekyan, V. G. Artyushenko, K. I. Kalaidzhyan, M. M. Mirakyan, and I. L. Pyl'nov, "Bending loss of hollow metal waveguides for mid-infrared range," Sov. Tech. Phys. Lett. 15, 602-603 (1989).

9. S. Karasawa, M. Miyagi, T. Nakamura, and H. Ishikawa, "Fab- rication of dielectric-coated rectangular hollow waveguides for $\mathrm{CO}_{2}$ laser light transmission," Trans. Inst. Electron. Inf. Commun. Eng. C-I J72, 637-641 (1989).

10. S. V. Azizbekyan, V. G. Artyushenko, E. M. Dianov, K. I. Kalaidzhyan, and M. M. Mirakyan, "Transmission of hollow metal waveguides in the mid-infrared region," Sov. Phys. Tech. Phys. 35, 196-198 (1990).

11. V. G. Artyushenko, K. I. Kalaidzhyan, and M. M. Mirakyan, "Flexible hollow waveguides for the mid-IR range," Sov. Phys. Tech. Phys. 36, 46-49 (1991).

12. H. Machida, H. Ishikawa, and M. Miyagi, "Low-loss lead fluoride-coated square waveguide for $\mathrm{CO}_{2}$ laser light transmission," Electron. Lett. 27, 2068-2070 (1991).

13. Y. Matsuura and M. Miyagi, "Bending losses and beam profiles of zinc selenide-coated silver waveguides for carbon dioxide laser light," Appl. Opt. 31, 6441-6445 (1992).

14. H. Machida, Y. Matsuura, H. Ishikawa, and M. Miyagi, "Transmission properties of rectangular hollow waveguides for $\mathrm{CO}_{2}$ laser light," Appl. Opt. 31, 7616-7622 (1992).

15. Y. Matsuura and M. Miyagi, "Er:YAG, CO, and $\mathrm{CO}_{2}$ laser delivery by ZnS-coated Ag hollow waveguides," Appl. Opt. 32, 6598-6601 (1993).

16. T. Abel, J. Hirsch, and J. A. Harrington, "Hollow glass waveguides for broadband infrared transmission," Opt. Lett. 19, 1034-1036 (1994).

17. Y. Matsuura, T. Abel, J. Hirsch, and J. A. Harrington, "Smallbore hollow waveguide for delivery of near singlemode IR laser radiation," Electron. Lett. 30, 1688-1690 (1994).

18. R. K. Nubling and J. A. Harrington, "Hollow-waveguide delivery systems for high-power, industrial $\mathrm{CO}_{2}$ lasers," Appl. Opt. 35, 372-380 (1996)

19. D. Su, S. Somkuarnpanit, D. R. Hall, and J. D. C. Jones, "Thermal effects in a hollow waveguide beam launch for $\mathrm{CO}_{2}$ laser power delivery," Appl. Opt. 35, 4787-4789 (1996).

20. J. Dal and J. A. Harrington, "High-peak-power, pulsed $\mathrm{CO}_{2}$ laser light delivery by hollow glass waveguides," Appl. Opt. 36, 5072-5077 (1997).

21. D. C. Chang and E. F. Kuester, "A hybrid method for paraxial beam propagation in multimode optical waveguides," IEEE Trans. Microwave Theory Tech. MTT-29, 923-933 (1981).

22. L. W. Casperson, "Gaussian beams in hollow metal waveguides," J. Opt. Soc. Am. A 17, 1115-1123 (2000).

23. M. Ghita and L. W. Casperson, "Gaussian beams in hollow metal waveguides: experiment," Appl. Opt. 40, 5459-5462 (2001).

24. L. A. Rivlin and V. S. Shul'dyaev, "Multimode waveguides for coherent light," Izv. Vyssh. Uchebn. Zaved. Radiofiz. 11, 572578 (1968).

25. E. E. Grigor'eva and A. T. Semenov, "Waveguide image transmission in coherent light (review)," Sov. J. Quantum Electron. 8, 1063-1073 (1978).

26. L. W. Casperson, "Gaussian light beams in inhomogeneous media,” Appl. Opt. 12, 2434-2441 (1973).

27. I. S. Gradshteyn and I. M. Ryzhik, Table of Integrals, Series, and Products, 4th ed. (Academic, New York, 1965), Eq. (3.896$1)$. 